\title{
Miteinander oder Nebeneinander?
}

Deutsche Juden und Nichtjuden im städtischen Alltag des späten 19. und frühen 20. Jahrhunderts

Vivre ensemble ou simplement côte-à-côte? La vie quotidienne des Juifs et nonJuifs allemands en milieu urbain entre la fin du XIXe et le début du XXe siècle Living together or in Parallel Societies? German Jews and Gentiles in Urban Everyday Life in the Late 19th and Early 20th Century

\section{Armin Owzar}

\section{OpenEdition}

Journals

Édition électronique

URL : https://journals.openedition.org/ceg/6854

DOI : $10.4000 /$ ceg. 6854

ISSN : 2605-8359

Éditeur

Presses Universitaires de Provence

Édition imprimée

Date de publication : 25 septembre 2019

Pagination : 23-37

ISBN : 979-10-320-0234-6

ISSN : 0751-4239

\section{Référence électronique}

Armin Owzar, "Miteinander oder Nebeneinander?", Cahiers d'Études Germaniques [Online], 77 | 2019,

Online erschienen am: 25 März 2021, abgerufen am 15 Juni 2021. URL: http://

journals.openedition.org/ceg/6854 ; DOI: https://doi.org/10.4000/ceg.6854 


\section{Miteinander oder Nebeneinander? \\ Deutsche Juden und Nichtjuden im städtischen Alltag des späten 19. und frühen 20. Jahrhunderts}

Armin OWZAR

Université Sorbonne Nouvelle - Paris 3 / CEREG (EA 4223)

Dass die Shoah den gesamten Verlauf der deutsch-jüdischen Geschichte überschattet, wird kaum jemand bezweifeln. Keine Einvernehmlichkeit besteht dagegen über die Länge und das Ausmaß dieses Schattens, insbesondere mit Blick auf die zweite Hälfte des, langen 19. Jahrhunderts', jenen Zeitraum, in dem neben den traditionellen Antijudaismus sukzessive ein biologistischer und eliminatorischer Rassen-Antisemitismus trat. Nach wie vor stellt sich die Frage, ob sich diese Jahrzehnte nur als Vorgeschichte der Shoah angemessen beschreiben lassen oder ob es nicht einer Historisierung bedarf, die jegliche teleologische Determiniertheit infrage stellt und eine offene Entwicklung postuliert? ${ }^{1}$

Will man darauf eine Antwort finden, gilt es, die Verbreitung und Akzeptanz exkludierender Ideologien, Maßnahmen und Verhaltensmuster zu bestimmen. Dabei sind drei methodologische Prämissen zu beachten. Erstens reicht es nicht aus, Exklusionsprozesse nur auf ideologischer oder politischer Ebene zu analysieren. Zweifelsohne sind solche Ansätze unverzichtbar. Insofern sich Exklusion aber primär auf der Ebene alltäglicher Beziehungen manifestiert: in der Schule und auf der Universität, in der Freizeit, beim Einkauf und am Arbeitsplatz, bedarf es auch eines Zugriffs, der nach der Verbreitung judenfeindlicher Stereotype im Alltag fragt und jenes alltägliche Verhalten in den Blick nimmt, das von den Betroffenen kaum reflektiert wurde, da die ihm zugrunde liegenden Normen längst internalisiert worden waren. Zweitens lässt sich eine Geschichte der Exklusion nicht schreiben, ohne dass man den Stellenwert ihres Komplementärs, der Inklusion, bestimmt - wobei Inklusion und Exklusion nicht nur einfach als zwei Seiten einer Medaille zu betrachten sind. Im Falle der deutsch-jüdischen Geschichte zwischen 1867 und 1933 waren sie vielmehr dialektisch aufeinander bezogen: Jeder Zugewinn an rechtlich-politischer Emanzipation und gesellschaftlicher Integration bewirkte eine Radikalisierung antijüdischer Programmatik und Ausgrenzung. Drittens ist ein vergleichender Zugriff, vor allem aus interkonfessioneller Perspektive, unverzichtbar. Nur wenn man die Gemeinsamkeiten und Unterschiede herausarbeitet, die den jüdisch-

1. Vgl. Moshe Zimmermann, Die deutschen Juden 1914-1945, München, Oldenbourg, 1997, S. XIII. 
nichtjüdischen und den katholisch-protestantischen Beziehungen zugrunde lagen, erkennt man, wie ,normal' religiöses Segregationsverhalten in den Jahrzehnten zwischen 1870 und 1930 war - aber auch welche Unterschiede zwischen Antikatholizismus, Antiprotestantismus und Antisemitismus bestanden.

Um diesen drei Prämissen gerecht zu werden, empfiehlt sich der Rückgriff auf ein vor allem in der Flüchtlingsforschung erprobtes Modell, das anhand dreier Indikatoren den Grad sozialer Nähe bzw. Ferne zwischen zwei Gruppen anzuzeigen vermag. ${ }^{2}$ Mithilfe des Indikators ,Kommerzium' werden die Wirtschaftsbeziehungen zwischen zwei Gruppen als Ausgangspunkt eines sich allmählich vollziehenden Integrationsprozesses untersucht. Demzufolge gibt die Bereitschaft zum regelmäßigen Austausch von Gütern, Geld und Dienstleistungen Auskunft über eine primär von ökonomisch-rationalen Interessen geleitete Intensität sozialer Beziehungen. Der zweite Indikator, ,Kommensalität', bezieht sich auf den gesellschaftlichen Verkehr: insbesondere auf jene Formen von Geselligkeit, wie sie in Vereinen, in Gasthäusern oder in privaten Räumlichkeiten praktiziert wird. Mithilfe dieses Indikators lässt sich der Grad emotionaler Integration messen. Eine Vergemeinschaftung aller Beteiligten liegt allerdings erst dann vor, wenn zwischen den zu untersuchenden Gruppen keinerlei Heiratsschranken (mehr) bestehen. Diesen Grad intensivster Form sozialer Beziehungen beschreibt der Indikator ,Konnubium'. Nützlich erweist sich diese Integrationstrias vor allem bei der Anwendung auf Gesellschaften mit soziokulturell definierten Minderheiten. Allerdings erlaubt das Modell nur eine Annäherung und bedarf einer ergänzenden Untersuchung des Wohnverhaltens, das sowohl Ursache als auch Resultat segregativen Verhaltens sein kann, sowie jener Stereotype, die der jeweils anderen Gruppe entgegengebracht werden und vielfach den Ausgangspunkt exkludierender Verhaltensweisen bilden. ${ }^{3}$

In der Regel wird man denn auch davon ausgehen können, dass stereotypes Denken und soziales Verhalten in einem Interdependenz-Verhältnis stehen. So bildet stereotypes Denken vielfach den Ausgangspunkt exkludierender Verhaltensweisen. Wer gegenüber einer Fremdgruppe keine oder nur gering ausgeprägte Negativ-Stereotype hat, wird mit deren Angehörigen vermutlich schneller in Kontakt treten als jemand, der ihr Aversionen entgegenbringt oder grundsätzlich ablehnend gegenüber eingestellt ist. Vice versa haben empirische Untersuchungen zeigen können, dass negative kollektive HeteroStereotype infolge positiver Individualkontakte abgebaut werden können. ${ }^{4}$ Gleichwohl erlauben es solche Beobachtungen nicht, grundsätzlich einen kausalen Nexus zwischen stereotypem Denken und (nicht)diskriminierendem Verhalten anzunehmen. So stoßen wir immer wieder auf Nichtjuden, die trotz intensiver privater Kontakte zu Juden überzeugte, ja fanatische Antisemiten

2. Siehe Marion Frantzioch, Die Vertriebenen. Hemmnisse, Antriebskräfte und Wege ihrer Integration in der Bundesrepublik Deutschland, West-Berlin, Dietrich Reimer, 1987, S. 193-197 und 239-242.

3. ZurStereotypenforschung sieheetwa Hans Henning Hahn ( $\mathrm{Hg}$.$) , HistorischeStereotypenforschung.$ Methodische Überlegungen und empirische Befunde, Oldenburg, bis, 1995.

4. Siehe Hilde Weiss, Antisemitische Vorurteile in Österreich. Theoretische und empirische Analysen, Wien, Wilhelm Braunmüller, 1984, S. 79-82. 
waren. Verwiesen sei nur auf Wilhelm Marr, der mit einer Jüdin verheiratet war. ${ }^{5}$ Und selbst im Falle einer erfolgreich vollzogenen sozialen Integration ehemaliger Außenseiter ist es nicht ausgeschlossen, dass subtile Formen der Ausgrenzung, etwa in Form diskriminierender Heterostereotype, fortbestehen. Eine weithin vorurteilsfreie Einstellung gegenüber Fremdgruppen wiederum muss nicht automatisch private Kontakte zur Folge haben. Einstellungsmuster und alltägliches Verhalten sind ungeachtet aller Interdependenzen zwei relativ autonome Bereiche und müssen dementsprechend gesondert betrachtet werden. Von einer vollkommen gelungenen Integration wird man wohl erst dann sprechen können, wenn identitätsstiftende Merkmale physiognomischer, ethnischer oder sozialer Provenienz von der Mehrheitsgesellschaft nicht mehr als minderwertig klassifiziert oder gar nicht mehr wahrgenommen werden.

Für eine empirische Bestandsaufnahme empfiehlt sich in diesem Zusammenhang ein ideen- und diskursgeschichtlicher Zugriff, der nach der Verbreitung diskriminierender Stereotype fragt. Gerade bei einer Untersuchung der Unterschichten stößt ein solcher Zugriff allerdings schnell an seine Grenzen. So sagt die Auflage und Verbreitung antisemitischer Publikationen und Predigten nur bedingt etwas über die tatsächlich erfolgte Internalisierung antisemitischer Stereotype aus. Aber auch von den Wahlerfolgen oder Niederlagen antisemitischer Kandidaten lässt sich nur eingeschränkt auf eine antisemitische Verhaltenspraxis der Wählerschaft schließen. So mancher überzeugte Antisemit blieb den Liberalen oder gar der sozialdemokratischen Partei treu, während andere wiederum durch die Wahl eines antisemitischen Kandidaten nur ihre (diffuse) Unzufriedenheit mit den herrschenden Verhältnissen zum Ausdruck bringen wollten und weiterhin gute Geschäftsbeziehungen zu Juden unterhalten und "sogar in guter Nachbarschaft mit ihnen leben" konnten. ${ }^{6}$ Einen größeren Erkenntnisgewinn zur Radikalität und zum Stellenwert eines ,ganz normalen', alltäglichen Antisemitismus versprechen dagegen Ego-Dokumente sowie die sogenannten Vigilanzberichte der hamburgischen Polizei. Dabei handelt es sich um ein Korpus von rund 20.000 Berichten inkognito ermittelnder Polizisten, die zwischen 1892 und 1910 beinahe täglich ausschwärmten, um sich ein Bild von der allgemeinen politischen Stimmung in der Hansestadt zu machen - ein in der Überlieferungsgeschichte einmaliges Quellenkorpus für die Alltagsgeschichte des deutschen Kaiserreichs. ${ }^{7}$

5. Siehe Olaf Blaschke, Katholizismus und Antisemitismus im Deutschen Kaiserreich, Göttingen, Vandenhoeck \& Ruprecht, 1997, S. 229.

6. Paul W. Massing, Vorgeschichte des politischen Antisemitismus, Frankfurt a.M., EVA, 1986 [1949], S. 112.

7. Im Folgenden zitiert als StaatsA HH [Staatsarchiv Hamburg], 331-3, Politische Polizei, S 3930. Eine kleine Auswahl dieser Berichte wurde ediert von Richard J. Evans (Hrsg.), Kneipengespräche im Kaiserreich. Die Stimmungsberichte der Hamburger Politischen Polizei 1892-1914, Reinbek, Rowohlt, 1989 und Sielke Salomon, Patrick Wagner (Hrsg.) , „Ich erlauschte folgendes Gespräch: ....”. Mit Polizeispitzeln durch Eimsbütteler Kneipen der Jahrhundertwende, Hamburg, Galerie Morgenland / Geschichtswerkstatt Eimsbüttel, [1995]. 
Wenn im Folgenden mit dem wilhelminischen Hamburg eine zeitliche wie räumliche Eingrenzung vorgenommen wird, so liegen dem nicht nur überlieferungspraktische, sondern auch inhaltliche Erwägungen zugrunde. Mit Blick auf die jüdisch-nichtjüdischen Sozialbeziehungen erweist sich die wilhelminische Zeit einerseits als Vorgeschichte der Weimarer Zeit, andererseits treten aber auch die signifikanten Unterschiede hervor, insofern der gesellschaftliche Antisemitismus nach dem Ersten Weltkrieg verbreiteter war und viel radikaler auftrat als zuvor. ${ }^{8}$ Aufgrund ihrer besonders komplexen und differenzierten Sozialstruktur und der daraus resultierenden Interessenskonflikte eignet sich dabei vor allem die Großstadt als Untersuchungsort der modernen Gesellschaft. ${ }^{9}$ Das gilt umso mehr für die Hafenstadt Hamburg, die wie keine andere deutsche Metropole jener Zeit einen multikulturellen Mikrokosmos bildete $^{10}$ und neben Berlin, Breslau und Frankfurt am Main zu den Zentren jüdischen Lebens in Deutschland zählte. ${ }^{11}$ Auch wenn der prozentuale Anteil der hamburgischen Juden an der Gesamtbevölkerung im Untersuchungszeitraum kontinuierlich sinken sollte - von 4,1 Prozent (1871) auf 1,9 Prozent (1910) -, erhöhte sich doch die absolute Zahl im selben Zeitraum um mehr als ein Drittel (37,2 Prozent), was sich nicht zuletzt der Zuwanderung vor allem osteuropäischer Juden verdankte. ${ }^{12}$ Von einer homogenen jüdischen,Community' konnte mithin nicht die Rede sein, weder in ethnischer oder kultureller noch in sozialer Hinsicht. So zählte fast die Hälfte der in Hamburg lebenden Juden zur Unterschicht; 54 Prozent gehörten den Mittelschichten an, gerade einmal 0,2 Prozent der Oberschicht, darunter der Reeder Albert Ballin, der Bankier Max M. Warburg und die Warenhausbesitzer Henry und Berthold Heilbuth, die immer wieder zur

8. Vgl. den Forschungsüberblick von Christoph Nonn, Antisemitismus, Darmstadt, Wissenschaftliche Buchgesellschaft, 2008, S. 68 und $72 f$.

9. Für die christlich-jüdischen Beziehungen in der ländlichen Gesellschaft siehe Utz Jeggle, Judendörfer in Württemberg, Tübingen, Tübinger Vereinigung für Volkskunde, 1999; sowie die einschlägigen Beiträge in Monika Richarz, Reinhard Rürup (Hrsg.), Jüdisches Leben auf dem Lande. Studien zur deutsch-jüdischen Geschichte, Tübingen, Mohr Siebeck, 1997. Siehe auch Nebeneinander Miteinander Gegeneinander. Zur Koexistenz von Juden und Katholiken in Süddeutschland im 19. und 20. Jahrhundert, hrsg. vom Haus der Geschichte Baden-Württemberg, Gerlingen, Bleicher, 2002.

10. Vgl. Rolf Italiaander, Vielvölkerstadt. Hamburg und seine Nationalitäten, Düsseldorf, Droste, 1986. Allgemein zur Stadtgeschichte Hamburgs im deutschen Kaiserreich siehe Ekkehard Böhm, "Der Weg ins Deutsche Reich. 1860-1880", in Werner Jochmann, Hans-Dieter Loose (Hrsg.), Hamburg. Geschichte der Stadt und ihrer Bewohner, Bd. 1: Loose (Hrsg.), Von den Anfängen bis zur Reichsgründung, Hamburg, Hoffmann \& Campe, 1986, S. 491-539 sowie Jochmann, „Handelsmetropole des Deutschen Reiches", in Jochmann, Loose (Hrsg.), Hamburg, Bd. 2: Jochmann (Hrsg.), Vom Kaiserreich bis zur Gegenwart, Hamburg, Hoffmann \& Campe, 1986, S. 15129.

11. Siehe Arno Herzig, Jüdische Geschichte in Deutschland. Von den Anfängen bis zur Gegenwart, München, C. H. Beck, 1997, S. 191f. Allgemein zur Lage der Juden im deutschen Kaiserreich siehe Shulamit Volkov, Die Juden in Deutschland 1780-1918, München, Oldenbourg, 2000; Steven M. Lowenstein, Paul Mendes-Flohr, Peter Pulzer, Monika Richarz, Umstrittene Integration 1871-1918 (= Deutsch-jüdische Geschichte in der Neuzeit, Bd. 3), München, C. H. Beck, 1997.

12. Angaben nach Helga Krohn, Die Juden in Hamburg. Die politische, soziale und kulturelle Entwicklung einer jüdischen Großstadtgemeinde nach der Emanzipation 1848-1918, Hamburg, Hans Christians, 1974, S. 66. 
Zielscheibe antisemitischer Hetze wurden. Allerdings propagierte nur eine kleine, wenngleich wortstarke Minderheit einen modernen Rassenantisemitismus. Die ganz überwiegende Mehrheit sowohl des liberalen Establishments als auch der meist sozialdemokratisch orientierten Arbeiterschaft wollte vom Antisemitismus nichts wissen. Dementsprechend wachsam reagierte die Obrigkeit auf jegliche Form antisemitischer Agitation und ließ einschlägige Veranstaltungen überwachen, die einhergehend mit dem erst in den 1890er Jahren in Hamburg erstarkenden politischen Antisemitismus durchgeführt wurden. ${ }^{13}$ Insofern gilt für die Hansestadt auch mit Blick auf die jüdisch-nichtjüdischen Beziehungen das, was Richard J. Evans bereits im Allgemeinen festgehalten hat: „Hamburg hatte seine Besonderheiten, wie jede andere deutsche Stadt auch, aber es war nicht ,untypischer' als Berlin oder München, Frankfurt oder Leipzig". ${ }^{4}$

Integration durch Kommerzium, Kommensalität und Konnubium kann sich in einem urbanen Großraum nur unter bestimmten Bedingungen vollziehen. Es bedarf gewisser topographischer Voraussetzungen, damit überhaupt die Möglichkeit eines Kontakts gegeben ist. Dabei kommt der Verteilung der zu untersuchenden Gruppen auf die Stadtteile und Quartiere eine zentrale und komplexe Bedeutung zu. Denn einerseits prädisponiert die räumliche Verteilung soziokultureller Kollektive deren Wirtschafts-, Freizeit- und Heiratsverhalten in nicht unbeträchtlichem Maße. Andererseits ist sie selbst schon Produkt segregativen bzw. integrativen Verhaltens. Beides lässt sich am Beispiel Hamburgs gut nachvollziehen. So spiegelte sich die spezifische Sozialstruktur derjenigen hamburgischen Juden, die Mitglieder der Israelitischen Gemeinde waren, auch in ihrer Verteilung auf das Stadtgebiet wider. Der signifikant hohe Anteil jüdischer Bürgerinnen und Bürger in den Mittelschichten hatte zur Folge, dass sie im Jahre 1885 sowohl in den Villenvierteln (in Harvestehude und in Rotherbaum) als auch im kleinbürgerlich geprägten Norden der Neustadt deutlich sowie in der südlichen Neustadt und in St. Pauli leicht überrepräsentiert waren. In den Arbeitervierteln lag ihr Anteil dagegen unterhalb des Durchschnitts; und in den ländlich geprägten Teilen des Stadtgebietes waren sie kaum oder gar nicht vertreten woran sich auch im Laufe der Weimarer Republik nur wenig ändern sollte. Dass nicht nur soziale, sondern auch religiöse Faktoren das jüdische Wohnverhalten bestimmten, wird deutlich, wenn man deren Verteilung innerhalb eines Viertels betrachtet. So lebten viele, meist kleinbürgerliche Juden um den in der Neustadt gelegenen und im Volksmund,Judenbörse' genannten Straßenmarkt. Orthodoxe Juden wiederum siedelten bevorzugt in Rotherbaum, im Gebiet des Grindels. In diesem von den Einwohnern ,Klein-Jerusalem' genannten Viertel, das noch 1928 von manchen Juden als "freiwilliges Ghetto" bezeichnet wurde, gab es nicht nur koschere Fleisch- und Geflügelhandlungen, sondern seit 1906 auch eine Synagoge (die Hauptsynagoge am Bornplatz) und seit 1911 eine Talmud ToraSchule (im Grindelhof). Liberale und religiös indifferente Juden bevorzugten

13. Siehe Kurt-Gerhard Riquarts, Der Antisemitismus als politische Partei in Schleswig-Holstein und Hamburg 1871-1914, Kiel, [Diss.], 1975, S. 182-287.

14. Richard J. Evans, „Einleitung“, in Evans (Hrsg.), Kneipengespräche im Kaiserreich, S. 7-39, hier S. 35. 
dagegen, je nach Einkommen und Vermögen, Harvestehude oder die westlichen Vororte. Mit sozial eher kleinbürgerlich strukturierten Vierteln wie Eimsbüttel gab es aber durchaus Stadtteile, in denen nicht nur Arbeiter, Handwerker, Kaufleute und Angestellte, sondern auch Protestanten, Katholiken und Juden sowie Sozialdemokraten und Antisemiten Block an Block, wenn nicht sogar Tür an Tür miteinander lebten. ${ }^{15}$ Dementsprechend stößt man hier auf zahlreiche höchst unterschiedliche Einstellungsmuster gegenüber Juden.

Stereotype und Ressentiments gegenüber Angehörigen anderer konfessioneller oder religiöser Gemeinschaften waren im Deutschland des 19. und 20 Jahrhunderts weitverbreitet. ${ }^{16}$ Beispielsweise existierte ein umfangreicher Fundus antikatholischer Urteile politischer, kultureller oder sozialer Provenienz, die allesamt einen konfessionsspezifischen Referenzpunkt aufwiesen. So stellten Protestanten einen Zusammenhang her zwischen dem angeblich mangelnden Patriotismus der Katholiken und deren Anhänglichkeit an den Papst im fernen Rom, dessen Worten eine höhere Autorität beigemessen werde als denen des Kaisers. Die Ende des 19. Jahrhunderts von Katholiken immer wieder beklagte Imparität in den staatlichen Funktionseliten und deren signifikant geringeren Anteil im höheren Bildungswesen führten sie auf eine aus dem katholischen Glauben resultierende Bildungsferne und eine vom Vatikan verordnete grundsätzliche Feindschaft gegenüber der Moderne zurück. Die mit Bezug auf Kriminalitätsstatistiken behauptete signifikante Überrepräsentanz von Katholiken bei Diebstahl, Raub und Mord erklärte man sich mit der besonderen Verbundenheit der Katholiken mit ihrer Kirche und ihrer religiösen Praxis, da durch die Möglichkeit des Beichtens die Hemmschwelle für Straftaten gesenkt werde. ${ }^{17}$

Was diesen bisweilen sogar militant ausgetragenen Antikatholizismus ${ }^{18}$ vom Antisemitismus, wie er seinerzeit auch in Hamburg u.a. von Hermann Ahlwardt propagiert wurde, fundamental unterschied, war der vollständige Verzicht auf biologische Herleitungen sowie jedwede Forderung nach einer eliminatorischen

15. Siehe hierzu das Statistische Handbuch für den Hamburgischen Staat 4, hrsg. vom Statistischen Bureau der Steuer-Deputation, Hamburg, Otto Meissner, 1891, S. 29; Ina Lorenz, "',Ahasver geht nach Eppendorf' - Zur Stadtteilkonzentration der Hamburger Juden im 19. und 20. Jahrhundert", Informationen zur modernen Stadtgeschichte 1, 1987, S. 23-28; Richard J. Evans, Tod in Hamburg. Stadt, Gesellschaft und Politik in den Cholera-Jahren 1830-1910, Reinbek, Rowohlt, 1996, S. 490f.; Ina Lorenz, „Die jüdische Gemeinde Hamburg 1860-1943. Kaiserreich - Weimarer Republik - NSStaat", in Arno Herzig (Hrsg.) in Zusammenarbeit mit Saskia Rohde, Die Juden in Hamburg 1590 bis 1990. Wissenschaftliche Beiträge der Universität Hamburg zur Ausstellung „Vierhundert Jahre Juden in Hamburg", Hamburg, Dölling und Galitz, 1991, S. 77-100, hier S. 81.

16. Siehe Christel Köhle-Hezinger, Evangelisch - katholisch. Untersuchungen zu konfessionellem Vorurteil und Konflikt im 19. und 20. Jahrhundert vornehmlich am Beispiel Württembergs, Tübingen, [Diss.], 1976.

17. Siehe Armin Owzar, „Ein Kampf der Kulturen? Intrakonfessionelle Auseinandersetzungen und interkonfessionelle Konflikte im deutschen Kaiserreich", Zeitschrift für Kirchengeschichte 116, 2005, S. 354-377.

18. Siehe dazu grundsätzlich Manuel Borutta, Antikatholizismus. Deutschland und Italien im Zeitalter der europäischen Kulturkämpfe, Göttingen, Vandenhoeck \& Ruprecht, 2010; Michael B. Gross, The War Against Catholicism. Liberalism and the Anti-Catholic Imagination in Nineteenth-Century Germany, Ann Arbor, The University of Michigan Press, 2004. 
Politik. Damit waren dem Antikatholizismus von Anfang an Grenzen gesetzt, derweil der Antisemitismus im Laufe des 19. Jahrhunderts einer Radikalisierung unterlag. Trotz aller Binnendifferenzen entstand so ein ideologischer Rahmen, in den sich sämtliche gebundene wie ungebundene Antisemitismen, auch die nichtrassistischen und nicht-eliminatorischen Varianten, wie sie etwa im katholischen oder nationalprotestantischen Deutschland auftraten, fassen ließen. ${ }^{19}$

Selbst in der sozialdemokratisch gesinnten oder organisierten Arbeiterschaft kursierten bisweilen antijüdische Stereotype und Ressentiments - ungeachtet der von derSozialdemokratieseitAufkommen derStoecker-Bewegung kompromisslos vorgenommenen Distanzierung vom politischen und gesellschaftlichen Antisemitismus in tagespolitischen Auseinandersetzungen und theoretischen Debatten. Zwar verurteilten die Sozialdemokraten den Antisemitismus als „Verschleierungs- und Ablenkungsstrategie unter dem Schein des AntiKapitalismus". ${ }^{20}$ Gleichzeitig aber versuchten sie, neben der Arbeiterschaft auch Volksschullehrer, kleine Handwerker und Geschäftsleute zu gewinnen - ebenjene Klientel, die auch von den antisemitischen Parteien umworben wurde. Und dabei schreckten sie mitunter vor solchen antisemitischen Stereotypen nicht zurück, die den Juden als Prototypen des modernen Kapitalisten diskriminierten - so wie sie bereits von Karl Marx in seinem Beitrag Zur Judenfrage formuliert worden waren. ${ }^{21}$ Propagiert wurden solche Stereotype weniger in Reden als in Karikaturen der sozialdemokratischen Tages- und Unterhaltungspresse, auf denen ,der jüdische Kapitalist' nicht nur durch bestimmte Kleidungsstücke (wie Smoking oder Zylinder) kenntlich gemacht wurde, sondern ihm auch typisch jüdisch klingende Namen (wie Moses, Levi oder Cohn) und bestimmte physische Merkmale (wie krumme Beine, schwarzes Haar, Knoll- oder Hakennase) zugeschrieben wurden. ${ }^{22}$ Dass ein solcher antisemitischer Antikapitalismus in Teilen der hamburgischen Arbeiterschaft verbreitet war, ist den Vigilanzberichten der Hamburger Polizei zu entnehmen. So zitiert Schutzmann Jochum einen Sozialdemokraten, der Folgendes in einem Gespräch am 22. April 1893 gegenüber einigen Antisemiten in der Kellerwirtschaft von Fischer (Grindelallee 86 ) verlauten ließ:

Ihr Antisemiten ihr seit die weißen Juden, und ihr seit noch viel schlechter, wie die echten schwarzen Juden. Ist ein Christ in Verlegenheit und geht zu einem schwarzen Juden, der hilft ihm aus der Verlegenheit. Natürlich zieht er seine Prozente davon, aber unter Wissen und Willen der Christen. Kommt aber ein Christ zu euch weiße Juden, so wird ihm so leicht nicht

19. Siehe dazu grundsätzlich Helmut Berding, Moderner Antisemitismus in Deutschland, Frankfurt a.M., Suhrkamp, 1988, S. 86-164.

20. Thomas Nipperdey, Deutsche Geschichte 1866-1918, Bd. 2: Machtstaat vor der Demokratie, München, C. H. Beck, 1992, S. 307.

21. Karl Marx, „Zur Judenfrage" [1844], in Karl Marx, Werke - Artikel - Entwürfe. März 1843 bis August 1844. Text (= Marx, Friedrich Engels, Gesamtausgabe [MEGA], Abt. 1, Bd. 2, hrsg. vom Institut für ML beim ZK der KPdSU und vom Institut für ML beim ZK der SED), Ost-Berlin, Dietz, 1982, S. 141169.

22. Für zahlreiche Belege siehe Rosemarie Leuschen-Seppel, Sozialdemokratie und Antisemitismus im Kaiserreich. Die Auseinandersetzungen der Partei mit den konservativen und völkischen Strömungen des Antisemitismus 1871-1914, Bonn, Neue Gesellschaft, 1978, insbesondere die im Anhang abgedruckten Quellen, S. 291-316. 
geholfen, helft ihr ihm, so wollt ihr auch eure Prozente davon ziehen, aber ohne Wissen und Willen der Geholfenen. So kommt ihr nachher auf die Anklagebank, denn ihr seit zu dumm für ein solches Geschäft zu machen. ${ }^{23}$

Solche Reden waren zumindest in Teilen der hamburgischen Arbeiterschaft nicht ungewöhnlich. Zudem kam es unter den einfachen Hafenarbeitern, aber auch bei sozialdemokratischen Journalisten und Gewerkschafter immer wieder zu antisemitischen Ausfällen gegenüber besonders verhassten Unternehmern wie dem Generaldirektor der HAPAG, Albert Ballin. ${ }^{24}$ Nichtsdestoweniger bleibt festzuhalten, dass die überwiegende Mehrheit der Arbeiter rassistische Diskriminierungen kategorisch ablehnte und selbst antisemitisch gesinnte Sozialdemokraten nicht der sogenannten Judenfrage, sondern der Verbesserung der Arbeitsbedingungen, der Veränderung ihrer sozialen Lage oder der Umgestaltung der Eigentumsverhältnisse Priorität einräumten.

Dementsprechend stößt man bei ihnen kaum auf Forderungen zum Boykott jüdischer Geschäfte. Nicht, dass es solche Forderungen nicht gegeben hätte. So votierte in einem am 21. März 1895 geführten Kneipengespräch ein sozialdemokratisch gesinnter Arbeiter für eine judenfeindliche Ausrichtung seiner Partei:

\begin{abstract}
Wenn man die ganzen Kapitalisten näher betrachten würde, so müßte man auch sehen, daß die größten und reichsten Kapitalisten Juden seien, die doch gewiß keine Produktion erzeugen würden. [...] Etwas von dem Antisemitismus müßte die sozialdemokratische Partei auch in ihr Programm mit aufnehmen, dann hätten wir hier in Hamburg nur eine einzige Partei. $^{25}$
\end{abstract}

Seine beiden Gesprächspartner vertraten indes ganz andere Standpunkte. Der eine, der offensichtlich dem reformistischen Flügel nahestand, sah mit der

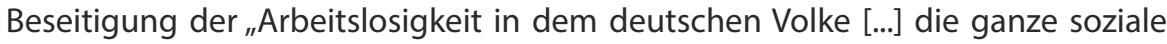
Frage gelöst" und sprach sich dafür aus, die Arbeitszeit zu verkürzen und die Akkordarbeit abzuschaffen. Der andere, der vermutlich dem revolutionären Flügel angehörte, befürwortete eine Auswanderung aller Kapitalisten. ${ }^{26}$ Mit einer Boykottforderung ausschließlich jüdischer Geschäfte konnten die beiden ebenso wenig anfangen wie die Mehrheit der hamburgischen Arbeiter, von denen viele in den preiswerten Warenhäusern von Heilbuth undTietzeinzukaufen pflegten. Wenn überhaupt machte man sich im sozialdemokratischen Milieu darüber Gedanken, wie man sich durch die Errichtung sogenannter Konsumgenossenschaften vom kapitalistisch organisierten Einzelhandel emanzipieren konnte. ${ }^{27}$ So ließ der im Jahre 1899 gegründete und von Sozialdemokraten getragene Konsum-, Bau-

23. Vigilanzbericht von Schutzmann Jochum vom 22. April 1893, in StaatsA HH, 331-3, Politische Polizei, S 3930-4, Bd. 1.

24. Siehe Michael Grüttner, Arbeitswelt an der Wasserkante. Sozialgeschichte der Hamburger Hafenarbeiter 1886-1914, Göttingen, Vandenhoeck \& Ruprecht, 1984, S. $208 f$. und 303.

25. Vigilanzbericht von Schutzmann Jochum vom 22. März 1895, in StaatsA HH, Politische Polizei, S 3930-4, Bd. 3.

26. Ibid.

27. Siehe Evans' Kommentar zum Kapitel Mittelstandspolitik und Antisemitismus in Evans (Hrsg.), Kneipengespräche im Kaiserreich, S. 302-306, hier 304. 
und Sparverein ,Produktion' zunächst im äußeren Hammerbrook, später dann in Barmbeck und anderen Stadtteilen verschiedene Wohnhäuser errichten, die mit einem Versammlungsraum, einer Kaffeerösterei, einem Restaurant und einer Verkaufsstelle eine Art Selbstversorgungseinheit bildeten. Außerhalb der Schul- und Arbeitszeit konnten ganze Familien nun unter sich bleiben; Einkaufen, Essen und Trinken, Politisieren und Feiern erfolgte hier unter einem Dach. ${ }^{28}$ Insofern trugen die Konsumgenossenschaften und Arbeitersiedlungen zur dezidierten Förderung eines segregrativen Konsum- und Freizeitverhaltens bei. Antisemitische Absichten lagen diesem Projekt indes keine zugrunde. In den Häusern lebten schließlich auch jüdische Arbeiter.

Sehr viel populärer waren Boykottforderungen dagegen im gewerblichen Mittelstand. Immer wieder begegnen einem um 1900 Kleinhändler, Handwerker und Lehrer, die, von Sozialneid und Konkurrenzangst erfüllt, sich gegen einen weiteren Zuzug osteuropäischer Juden oder für einen Boykott jüdischer Warenhäuser aussprachen. Gelegentlich wurde auch die Forderung nach einer Vertreibung jüdischer Geschäftsleute erhoben, so etwa von jenen sechs Geschäftsleuten, die am 7. Februar 1895 in der Wirtschaft von Winkelholz (in der Eimsbütteler Straße 15) belauscht wurden. Dem Bericht des Schutzmanns lässt sich entnehmen, dass sie sich eine Wirtschaftskrise geradezu herbeiwünschten, um ihre mehrheitlich liberal gesinnten Kollegen für eine antisemitische Politik zu gewinnen:

\footnotetext{
Die Geschäftslage des Mittelstandes müßte hier in Hamburg immer noch schlechter durch die Juden gemacht werden, wie sie gegenwärtig sei, damit auch die Geschäftsleute, welche bis jetzt noch dem Liberalismus angehören, zur richtigen Einsicht kommen würden. [...] Sie [die Geschäftsleute] können und dürfen hier in Hamburg fest hoffen, daß der Mittelstand bald bessere Tage sehen werde, wenn der letzte Jude über die Grenze gebracht sei. ${ }^{29}$
}

Ein Sprachrohr fanden solche Judenhasser in den Deutsch-sozialen Blättern, die im Oktober 1892 eine Artikelserie unter dem Titel Hamburg an der Elbe. Eine jüdisch-patrizische Aktien-Gesellschaft veröffentlichten, in der Front gegen die Symbiose christlicher und jüdischer Geschäftsleute gemacht wurde. ${ }^{30}$

In der hamburgischen Oberschicht stießen solche Verlautbarungen kaum auf Zustimmung. Nur sehr selten finden sich in den Quellen Belege für antijüdische Ressentiments oder gar einen offen zur Schau getragenen Antisemitismus. Nicht dass es unter den Patriziern keine Vorbehalte gegenüber Juden gegeben hätte. ${ }^{31}$ Insgesamt aber war auf der Ebene der politischen und gesellschaftlichen

28. Siehe Matthias Judt, „Vom Genossen zum Aktionär. Zur Geschichte der Hamburger Konsumgenossenschaft ,Produktion'", Hamburger Wirtschafts-Chronik NF, 2, 2001-2002, S. 197235.

29. Vigilanzbericht von Schutzmann Jochum vom 8. Februar 1895, in StaatsA HH, Politische Polizei, S 3930-4, Bd. 3.

30. Tesdorpf, "Hamburg an der Elbe. Eine jüdisch-patrizische Aktien-Gesellschaft", Deutsch-Soziale Blätter 7, Nr. 218 (16. Oktober 1892), S. 491f., Nr. 219 (23. Oktober 1892), S. 505f. und Nr. 220 (30. Oktober 1892), S. $516 f$.

31. Siehe Erika Hirsch, Jüdisches Vereinsleben in Hamburg bis zum Ersten Weltkrieg. Jüdisches Selbstverständnis zwischen Antisemitismus und Assimilation, Frankfurt a.M. u.a., Peter Lang, 
Kontakte der Integrationsprozess relativ weit vorangeschritten - wenn auch nicht abgeschlossen. So gab es nach wie vor keinen jüdischen Hamburger auf einem Senatorenposten; dafür aber waren Juden sowohl in der hohen Verwaltung als auch in der Bürgerschaft mit zum Teil hohen Positionen vertreten. Verwiesen sei nur auf Albert Wolffson, der es bis zum Vorsitzenden der Fraktion der Rechten brachte. ${ }^{32}$

Dass es sich dabei nur um eine Teilintegration handelte, wird deutlich, wenn man den Indikator Kommensalität in den Blick nimmt. Insbesondere im Vereinswesen spiegelt sich die starke Segregation von Juden und Nichtjuden. So gab es allein in Hamburg innerhalb des Untersuchungszeitraums 146 jüdische Vereine, darunter Jugend- und Frauenvereine, Sport- und Gesangsvereine, Spar-, Berufs- und Wohltätigkeitsvereine. ${ }^{33} \mathrm{Im}$ Rückblick lässt sich nicht eindeutig entscheiden, zu welchen Anteilen sich dieses Phänomen der Ausgrenzung, etwa durch die informelle Einführung eines Arier-Paragraphen, oder der (infolge der Milieubildung auch bei Katholiken und Sozialdemokraten zu beobachtenden) Selbstisolation verdankte, ob die in jüdischen Vereinen organisierten Hamburger prinzipiell keinen Kontakt zu Nichtjuden unterhalten oder nur potentiellen Diskriminierungen präventiv zuvorkommen wollten. Vieles spricht dafür, dass hierbei die soziale Zugehörigkeit eine nicht geringe Rolle spielte und die mehr oder weniger freiwillig vollzogene Segregation vor allem in den unteren Mittelschichten besonders stark ausgeprägt war. Das lässt sich etwa an der Entwicklung des hamburgischen Logenwesens ablesen. Bereits seit den 1840er Jahren war man dazu übergangen, auch Juden aufzunehmen und das mit steigender Tendenz. Mit 31 Prozent erreichte der Anteil jüdischer Brüder in den liberalen Logen im Jahre 1890 seinen Höhepunkt, um dann seit der Jahrhundertwende wieder zu sinken (auf 24,4 Prozent im Jahre 1910) - derweil die Mitgliederzahl des 1887 gegründeten Ablegers des B'nai B'rith-Ordens, der sich verstärkt aus den unteren Mittelschichten rekrutierte, kontinuierlich anstieg. ${ }^{34}$

Einen solchen Segregationstrend beobachtet man auch in anderen während der Freizeit genutzten Räumen. So überdauerte die kaum überwindbare "Kluft zwischen demjüdischen und dem nichtjüdischen Bürgertum “ ${ }^{35}$, wiesiein Hamburg schon zur Zeit der Reichsgründung bestanden hatte, vor allem bei Soiréen oder Essenseinladungen bis zum Ersten Weltkrieg. Und auch hier korrespondierte der allgegenwärtigen Ausgrenzung ein Trend zunehmender Selbstisolation - und das nicht nur bei zionistisch eingestellten Juden. Symptomatisch dafür ist ein Aufruf, den der Deutsch-Israelitische Gemeindebund über den Centralverein deutscher Staatsbürger jüdischen Glaubens verbreiten ließ:

1996, S. 21

32. Siehe Evans, Tod in Hamburg, S. 490f.

33. Für ein Verzeichnis der in Hamburg vertretenen jüdischen Vereine siehe Hirsch, Jüdisches Vereinsleben in Hamburg bis zum Ersten Weltkrieg, S. 375-380.

34. Siehe Stefan-Ludwig Hoffmann, Die Politik der Geselligkeit. Freimaurerlogen in der deutschen Bürgergesellschaft 1840-1918, Göttingen, Vandenhoeck \& Ruprecht, 2000, S. 77, 123 und $197 f$.

35. Jochmann, „Handelsmetropole des Deutschen Reiches", S. 58. 
Halten wir treue Freundschaft mit bewährten christlichen Freunden; aber meiden wir leichtfertigen, leichtsinnigen Umgang; meiden wir die gesellschaftliche Berührung mit Judenfeinden; beschränken wir uns nicht auf den Umgang mit Glaubensgenossen, aber weichen wir ihm auch nicht aus. [...] [S] uchen wir Bildung, Belehrung, Aufklärung zu fördern, zunächst und vorzugsweise in den Kreisen der Glaubensgenossen. [...] Hüten wir uns aber vor Zudringlichkeit auch in geselliger Beziehung. ${ }^{36}$

Auch in diesem Fall haben wir es mit einer Willensbekundung zu tun. Zuverlässige Angaben über die Zahl derer, die solchen Empfehlungen folgten, liegen nicht vor. Immerhin bestätigen einige Selbstzeugnisse, dass private Kontakte zwischen Juden und Nichtjuden innerhalb der hamburgischen Mittelschichten eher die Ausnahme bildeten und die Kinder der wohlangesehenen Hausherren und Familienväter in ihrer Freizeit, bei Spielen, Vorträgen und Tanzveranstaltungen, zumeist unter sich blieben. ${ }^{37}$

Selbst den Angehörigen der deutsch-jüdischen Wirtschaftselite blieb eine vollständige Integration ins gesellschaftliche Leben der Hansestadt versagt. Die hamburgischen Juden bildeten eine Subkultur - so wie in allen deutschen Städten, wo sich der „persönliche gesellige und freundschaftliche Umgang [...] im wesentlichen auf die jüdischen Kreise" beschränkte und darüber hinausgehende Kontakte weniger zu gleichgestellten Angehörigen des Großbürgertums als vielmehrzu Angehörigen derMittelschichten, namentlichzum Bildungsbürgertum bestanden. ${ }^{38}$ Offensichtlich, so der zeitgenössische Beobachter Gustav Schiefler, galt es "nicht für ,fein"', einem geselligen Kreis mit vielen Juden anzugehören. ${ }^{39}$ Auch der in Hamburg seit 1874 als Senatssekretär arbeitende Julius von Eckardt wusste zu berichten, dass sich das Althamburgertum "trotz entschiedener Judenfreundlichkeit“ in „,sozialer Rücksicht gegen das jüdische Element “ abschloss und "von verschwindenden Ausnahmen abgesehen, [...] auch die reichen und angesehenen Juden außerhalb der ,Gesellschaft" standen. Sie bildeten, so von Eckardt, „einerlei ob getauft oder nicht getauft, eine Welt für sich, die sich - wie allenthalben - durch geistige Regsamkeit und lebhafteste Empfänglichkeit für allgemeine Interessen auszeichnete". 40

Kein Fall illustriert dies besser als der des Großreeders Albert Ballin. Obwohl einer der wohlhabendsten Männer der Hansestadt zählte auch er zu seinen Freunden fast nur "Männer seines Glaubens", darunter den Unternehmer Emil Rathenau sowie die Bankiers Carl Fürstenberg und Max M. Warburg ${ }^{41}$, dessen

36. Empfehlung des Centralvereins deutscher Staatsbürger jüdischen Glaubens [o.J.], zitiert nach Krohn, Die Juden in Hamburg, S. 207.

37. Siehe etwa Dora Lehmann, Erinnerungen einer Altonaerin 1866-1946, hrsg. vom Joseph Carlebach Institut, Hamburg, Dölling und Galitz, 1998, S. 11-98, insbesondere S. 78.

38. Trude Maurer, Die Entwicklung der jüdischen Minderheit in Deutschland (1780-1933). Neuere Forschungen und offene Fragen, Tübingen, Max Niemeyer (IASL. Sonderheft 4) 1992, S. 165.

39. Gustav Schiefler, Eine Hamburgische Kulturgeschichte 1890-1920. Beobachtungen eines Zeitgenossen, bearb. von Gerhard Ahrens, Hans-Wilhelm Eckard und Renate Hauschild-Thiesen, Hamburg, Verein für Hamburgische Geschichte, 1985, S. 281.

40. Julius von Eckardt, Lebenserinnerungen, Bd. 1, Leipzig, Hirzel, 1910, S. 204.

41. Peter Franz Stubmann, Ballin. Leben und Werk eines deutschen Reeders, Berlin, Hermann Klemm, 1926, S. 174. 
Freundeskreis sich ebenfalls hauptsächlich aus Juden zusammensetzte. ${ }^{42}$ Schließlich konnte beiden nicht verborgen bleiben, dass die freundliche Distanz, mit der innen das hamburgische Patriziat begegnete, außerhalb der Stadtmauern die Ausnahme bildete. Vor allem der preußische Adel ließ Ballin und Warburg auf mehr oder weniger subtile Weise seine Verachtung spüren. Nicht einmal ihre so genannte Freundschaft zu Wilhelm II. öffnete den beiden sämtliche Türen zur guten Gesellschaft; selbst sie wurden nur höchst selten zu den feierlichen Berliner Hofsoiréen eingeladen. Vielmehr empörte sich ein "großer Teil der preußischen Aristokratie" über Wilhelms Verhältnis zu Ballin und bezeichnete diesen hinter vorgehaltener Hand als Parvenü oder gar auf rassistische Weise als "SEMI-Imperator". Anders als in England, wo Juden wie Ballin „Voll anerkannte und beliebte Mitglieder der begüterten [...] Gesellschaft" waren, betrachtete man die deutsch-jüdischen Größen der Finanz- und Industriewelt in Preußen lediglich als "Günstlinge des Königs" und nicht als "Angehörige des aristokratischen ,Establishments" ${ }^{\prime \prime \prime}{ }^{43}$ An dieser Abneigung des deutschen Adels gegenüber jüdischen Angehörigen der Oberschicht sollte sich auch nach dem Ende der Monarchie wenig ändern. Selbst diejenigen Teile des deutschen Adels, die sich nicht offen zum Antisemitismus bekannten, gingen auf Distanz zum Judentum. „Man pflegte Juden und Menschen jüdischer Abstammung aus dem Wege zu gehen, wo man es mit Anstand konnte", so Joachim von Dissow, der selbst einem uralten holsteinischen Geschlecht entstammte und freundschaftliche Kontakte zu jüdischen Kreisen unterhielt. Dem Adel, so erklärte es sich der Historiker, sei das Judentum „wegen seiner ausgeprägten Begabung für das Erwerbsleben wesensfremd, wegen seiner scharfen Intellektualität unheimlich und unsympathisch, und lächerlich wegen seiner Freude am sozialen Aufstieg" gewesen. ${ }^{44}$

Ganz anders gestalteten sich dagegen die jüdisch-nichtjüdischen Beziehungen in den Unterschichten Hamburgs. Trotz der bisweilen artikulierten Ressentiments gegenüber jüdischen Kapitalisten enthalten die Quellen keine Hinweise auf Berührungsängste oder gar feindselige Begegnungen jedweder Art. Zu antisemitischen Pöbeleien in Kaffeehäusern oder auf offener Straße, wie sie für die erste Hälfte des 19. Jahrhunderts in Hamburg überliefert sind ${ }^{45}$ und wie es sie andernorts auch um 1900 noch gab ${ }^{46}$, sollte es während des Kaiserreichs nicht mehr kommen. Auch Lokalverbote für jüdische Mitbürger sind nicht

42. Siehe Eduard Rosenbaum, A. J. Sherman, Das Bankhaus M. M. Warburg \& Co. 1798-1938, Hamburg, Hans Christians, 1976, S. 122 und 130.

43. Lamar Cecil, Albert Ballin. Wirtschaft und Politik im deutschen Kaiserreich 1888-1918, mit einer Einführung von Günther Jantzen, Hamburg, Hoffmann \& Campe, 1969, S. 96-98.

44. Joachim von Dissow, Adel im Übergang. Ein kritischer Standesgenosse berichtet aus Residenzen und Gutshäusern, Stuttgart, W. Kohlhammer, 1961, S. 184.

45. Siehe Evans, Tod in Hamburg, S. 491.

46. Siehe Christoph Nonn, Eine Stadt sucht einen Mörder. Gerücht, Gewalt und Antisemitismus im Kaiserreich, Göttingen, Vandenhoeck \& Ruprecht, 2002, S. 43-49; Helmut Walser Smith, Die Geschichte des Schlachters. Mord und Antisemitismus in einer deutschen Kleinstadt, Göttingen, Wallstein, 2002, S. 26-30, 38 und 46-55. 
bezeugt - im Gegensatz zu manchen Ost- und Nordseebädern, in denen Juden als unerwünschte Gäste galten. ${ }^{47}$

Allerdings liegt die Annahme nahe, dass die hamburgischen Juden jene Lokale der Hansestadt zu meiden pflegten, deren Gastwirte zum Antisemitismus übergetreten waren oder in denen antisemitische Parteien oder Vereine ihre Sitzungen abzuhalten pflegten. Und auch in einer normalen Wirtschaft mussten zumindest jene, die - ob jüdisch oder nicht - für Juden gehalten wurden, schon beim Betreten mit abfälligen Blicken oder abwertenden Bemerkungen rechnen. Darüber hinaus gab es auch in Hamburg orthodoxe oder traditionalistisch eingestellte Juden, die teils oder ganz an den rituellen Speisevorschriften festhielten oder auf den Ausschank koscheren Bieres und Weines bestanden - was den Besuch der allermeisten Gastwirtschaften nahezu unmöglich machte. Lokale mit koscherer Küche und koscherem Ausschank wiederum wurden von Nichtjuden nur selten besucht. In vielen meist kleineren Städten, wo es solche jüdischen Gastwirtschaften gar nicht gab, kehrten orthodoxe Juden oftmals bei einer jüdischen Familie ein, wo sie gegen Bezahlung ein koscheres Essen vorgesetzt bekamen. ${ }^{48}$ Diejenigen aber, die sich in eine allgemeine Gaststätte begaben, stießen hier sehr oft auf eine unsichtbare, mitten durch den Gastraum verlaufende Grenze, die die privilegierten und zumeist nichtjüdischen Gästen vorbehaltenen Sitzplätze am Stammtisch abtrennte. Während nicht nur für Großstädte, sondern auch Kleinstädte und Dörfer zahlreiche jüdisch-christliche Frauenstammtische bezeugt sind, waren jüdische Männer nur selten an einem Stammtisch geduldet; in der Regel wurde ihre "bloße Anwesenheit" von kleinstädtischen Honoratioren "als Provokation empfunden". 49

Es kann mithin kein Zweifel daran bestehen, dass die jüdisch-nichtjüdischen Beziehungen im wilhelminischen Deutschland durch Exklusion und Isolation, sei es aus Überzeugung, sei es zum Selbstschutz, gekennzeichnet waren - und das insbesondere in den städtischen Mittelschichten. ${ }^{50}$ In allen während der Freizeit genutzten Räumen: im Vereinswesen, auf Abendgesellschaften, in Gastwirtschaften und auch im Urlaub existierten unsichtbare Schranken, auch und sogar verstärkt in der Weimarer Republik ${ }^{51}$ - Schranken, wie sie, wenngleich in abgeschwächter Form, auch zwischen Protestanten und Katholiken mitunter bis

47. Siehe dazu ausführlich Frank Bajohr, „Unser Hotel ist judenfrei“. Bäder-Antisemitismus im 19. und 20. Jahrhundert, Frankfurt a.M., Fischer Taschenbuch, 2003.

48. Diesen Hinweis verdanke ich einer schriftlichen Auskunft der Mitbegründerin des Jüdischen Museums Westfalen in Dorsten Fr. Johanna Eichmann vom 5. Juni 2000.

49. Volker Ullrich, Die nervöse Großmacht. Aufstieg und Untergang des deutschen Kaiserreichs 18711918, Frankfurt a.M., S. Fischer, 1997, S. 393.

50. Für Schleswig-Holstein und Lübeck bestätigt diesen Befund Bettina Goldberg, Abseits der Metropolen. Die jüdische Minderheit in Schleswig-Holstein, Neumünster, Wachholtz, 2011, S. 241. Für Königsberg siehe Stefanie Schüler-Springorum, Die jüdische Minderheit in Königsberg/ Preußen, 1871-1945, Göttingen, Vandenhoeck \& Ruprecht, 1996, S. 81-86.

51. Vgl. Nonn, Antisemitismus, S. 66-74. Siehe auch Monika Richarz, Einführung, in Monika Richarz (Hrsg.), Jüdisches Leben in Deutschland. Selbstzeugnisse zur Sozialgeschichte 1918-1945, Stuttgart, DVA, 1982, S.13-73, hier S. 27. 
zu Beginn der 1960er bestanden. ${ }^{52}$ Allerdings gab es nicht nur ein Nebeneinander; es gab auch ein Miteinander. Die Schranken waren nicht immer so hoch und unüberwindlich, dass es überhaupt keinen geselligen Kontakt zwischen Juden und Nichtjuden gegeben hätte. ${ }^{53}$ In der hochgradig fragmentierten Gesellschaft des Kaiserreichs, in der Arbeiter und Bürger, Sozialdemokraten und Konservative in ihrer Freizeit nur selten miteinander ins Gespräch kamen ${ }^{54}$, boten sich Juden, Katholiken und Protestanten durchaus Räume, in denen sie miteinander verkehrten - zumindest wenn sie ein und derselben Schicht angehörten. Darüber hinaus existierten beim Militär, am Arbeitsplatz und in der Schule weitere Räume, in denen ungeachtet partieller Exklusion mileuübergreifende Kommunikation möglich war.

Dass der Integrationsprozess zumindest in Teilen der hamburgischen Mittelund Unterschichten voranschritt, zeigt auch eine Analyse des Heiratsverhaltens. Demzufolge nahm die Zahl der zwischen Juden und Nichtjuden geschlossenen Ehen hier kontinuierlich (und stärker als in anderen deutschen Städten) zu. ${ }^{55} \mathrm{Lag}$ der Anteil sogenannter Mischehen unter den hamburgischen Juden zwischen 1886 und 1890 noch bei 23,1 Prozent, so waren es zwischen 1906 und 1910 bereits 39,2 Prozent und 1933 gar 57,1 Prozent - wobei der Anteil jüdischer Männer, die einen nichtjüdischen Ehepartner heirateten, mit Ausnahme der Jahre von 1901 bis 1905 signifikant höher war als der jüdischer Frauen. ${ }^{56}$ Freilich sagen solche Zahlen nur etwas aus über die Integrationsbereitschaft jener jüdischen und christlichen Kreise, die einander ohnehin grundsätzlich aufgeschlossen begegneten. ${ }^{57}$ Ihnen standen diejenigen Katholiken, Protestanten und Juden gegenüber, die sich weiterhin abschotteten, die einen Partner desselben Bekenntnisses heirateten und ihre Kinder auf katholische, evangelische und jüdische Schulen schickten. Selbst

52. Ausführlich dazu Olaf Blaschke, „Das 19. Jahrhundert: Ein Zweites Konfessionelles Zeitalter?", Geschichte und Gesellschaft 26, 2000, S. 38-75; Olaf Blaschke (Hrsg.), Konfessionen im Konflikt. Deutschland zwischen 1800 und 1970: ein zweites konfessionelles Zeitalter, Göttingen, Vandenhoeck \& Ruprecht, 2002. Siehe auch Armin Owzar, „'Keine Lust' zur Diskussion. Zum Kommunikationsverhalten deutscher Katholiken, 1870 bis 1930", Schweizerische Zeitschrift für Religions- und Kirchengeschichte 101, 2007, S. 123-149.

53. Siehe Marion Kaplan, „Konsolidierung eines bürgerlichen Lebens im kaiserlichen Deutschland 1871-1918", in Kaplan (Hrsg.), Geschichte des jüdischen Alltags in Deutschland. Vom 17. Jahrhundert bis 1945, hrsg. im Auftrag des Leo Baeck Instituts, München, C. H. Beck, 2003, S. 225-344, hier S. 332; Till van Rahden, Juden und andere Breslauer. Die Beziehungen zwischen Juden, Protestanten und Katholiken in einer deutschen Großstadt von 1860 bis 1925, Göttingen, Vandenhoeck \& Ruprecht, 2000.

54. Siehe Armin Owzar, "Reden ist Silber, Schweigen ist Gold“. Konfliktmanagement im Alltag des wilhelminischen Obrigkeitsstaates, Konstanz, UVK, 2006.

55. Siehe Kerstin Meiring, Die Christlich-Jüdische Mischehe in Deutschland 1840-1933, Hamburg, Dölling und Galitz, 1998, insbesondere S. 81.

56. Errechnet nach Angaben bei Krohn, Die Juden in Hamburg, S. 69 und Baruch Z. Ophir, "Zur Geschichte der Hamburger Juden 1919-1939", in Peter Freimark (Hrsg.), Juden in Preußen Juden in Hamburg, Hamburg, Hans Christians, 1983, S. 81-97, hier S. 89.

57. Vgl. auch Nicola Wenge, Integration und Ausgrenzung in der städtischen Gesellschaft. Eine jüdisch-nichtjüdische Beziehungsgeschichte Kölns 1918-1933, Mainz, Philipp von Zabern, 2005, S. 93-115. 
in einer so offenen und von Liberalität gekennzeichneten Stadt wie Hamburg blieben diesen die Lebenswelten der jeweils Andersgläubigen verschlossen.

Aber auch dort, wo es zu mitunter intensiven Kontakten kam, erhielt sich oftmals eine gewisse Reserve. So gelangt auch Till van Rahden am Beispiel Breslaus zu dem Schluss, dass es angesichts der vielen Möglichkeiten, sich im Alltag kennenzulernen, weniger bemerkenswert sei, , daß es viele Freundschaften zwischen Juden einerseits und Protestanten und Katholiken andererseits gab, sondern daß es nicht noch mehr waren und in vielen dieser Freundschaften ein Rest der sozialen Distanz spürbar blieb." ${ }^{58}$ Diesem Phänomen einer sozialen Distanz ist empirisch mit der Integrationstrias kaum beizukommen. Über die subtilen Formen der Ausgrenzung und deren psychologische Folgen erfahren wir in den Quellen nur wenig. Eine Ausnahme bilden die Erinnerungen des jüdischen Journalisten Gustav Mayer, der, 1904 von Brüssel nach Hamburg zurückgekehrt, mit Unbehagen konstatierte, dass in der Hansestadt "Deutsche und [sic] Juden noch immer nebeneinander herlebten". Seine jüdische Herkunft, die für ihn allmählich in den Hintergrund getreten war, spielte auf einmal wieder eine besondere Rolle. Denn nicht nur die Antisemiten, sondern auch andere nichtjüdische Mitbürger betrachteten ihn weiterhin primär als Juden. Erst rückblickend, auf dem Hintergrund der Shoah, verstand Mayer, dass diese Form der Identifizierung in letzter Konsequenz sein Deutschsein infrage gestellt hatte. Mayer wörtlich: „Und wenn ich selbst voll Überzeugung ,wir Deutsche' sagte, hätte ich nicht schon immer schärfer darauf acht geben müssen, ob nicht um den Mund der anderen ein ironisches oder gar höhnisches Lächeln aufzuckte?"59

58. van Rahden, Juden und andere Breslauer, S. 132.

59. Gustav Mayer, Erinnerungen. Vom Journalisten zum Historiker der deutschen Arbeiterbewegung, Zürich/Wien, Europa, 1949, S. 149 und 364f. 